\title{
Preoperative prediction of Gleason grade in radical prostatectomy specimens: the influence of different Gleason grades from multiple positive biopsy sites
}

\author{
Chistopher K Poulos ${ }^{1}$, Joanne K Daggy² and Liang Cheng ${ }^{1}$ \\ ${ }^{1}$ Department of Pathology and Laboratory Medicine and ${ }^{2}$ Division of Biostatistics, Indiana University School \\ of Medicine, Indianapolis, IN, USA
}

\begin{abstract}
The Gleason score of prostate adenocarcinomas is an important preoperative predictor of cancer behavior, and is used to help guide treatment. In the setting of more than two positive biopsy sites, pathologists usually grade the tumor at each site separately, and the Gleason score may differ from each positive site. This study seeks to determine if the highest Gleason score in all biopsy sites, or the Gleason score in the site with the highest tumor volume on the needle biopsy is the best predictor of final Gleason score in the radical prostatectomy specimens. Various preoperative biopsy findings were analyzed. All 151 patients had at least two positive biopsy sites and underwent radical prostatectomy. Primary and secondary Gleason pattern grades were assigned for each positive biopsy site. The tumor volume in the needle biopsy site was defined by the percentage of areas of biopsy cores involved by cancer. The radical prostatectomy specimens were completely embedded and processed in the whole-mount method. The Gleason score from both the biopsy site with the highest Gleason score and the biopsy site with the highest tumor volume on the needle biopsy correlated equally well with final Gleason score at radical prostatectomy (Spearman correlation coefficient $=0.54$ for both, $P<0.001)$. The Gleason score from both the biopsy site with the highest Gleason score and the biopsy site with the highest tumor volume on the needle biopsy also correlated with primary Gleason pattern grade at radical prostatectomy (Spearman correlation coefficient $=0.53$ for both, $P<0.001$ ). Secondary Gleason pattern grade from the biopsy site with the highest tumor volume on the needle biopsy correlated with secondary Gleason pattern grade at radical prostatectomy slightly better than those from the biopsy site with the highest Gleason score (Spearman correlation coefficient, 0.32 vs 0.24 ; both $P<0.001$ ). Our data indicate that the highest Gleason score from all sites and the Gleason score from the site with the highest tumor volume on the needle biopsy are equally and significantly predictive of final Gleason score on radical prostatectomy. Both methods of prediction are significantly predictive of primary and secondary Gleason pattern grade on radical prostatectomy. We recommend that the highest Gleason score from all positive biopsy sites should be used when assigning an initial score using needle biopsies.
\end{abstract}

Modern Pathology (2005) 18, 228-234, advance online publication, 8 October 2004; doi:10.1038/modpathol.3800302

Keywords: prostatic neoplasm; Gleason grade; radical prostatectomy; needle biopsy; adenocarcinoma

The Gleason grading system is an architectural based grading system, and has become one of the most accepted and important predictors of the prognosis and behavior of prostate adenocarcinoma. ${ }^{1-8}$ In general, the Gleason score on 18-gauge

Correspondence: Dr L Cheng, MD, Department of Pathology and Laboratory Medicine, Indiana University Medical Center, University Hospital 3465, 550 North University Blvd., Indianapolis, IN 46202, USA.

E-mail: lcheng@iupui.edu

Received 22 June 2004; revised 27 August 2004; accepted 28 August 2004; published online 8 October 2004 needle biopsy specimens has been found to correlate well with the Gleason score on radical prostatectomy specimen and to have good interobserver reproducibility; however, the correlation is not perfect and under grading is a particular problem, in up to $58 \%$ of cases. ${ }^{1-3,6,9-14}$ Some have advocated not assigning biopsy scores of 2-4 to eliminate errors and potential harm to patients. ${ }^{10,11}$

Sextant biopsies have been routinely used by many urologists, and prostate cancer is often found in multiple biopsy sites from the same patient. Despite extensive studies on the predictive values of prostate biopsy Gleason scores for final Gleason 
scores at radical prostatectomy, the implications of different Gleason scores from more than one site are uncertain despite the recommendation of assigning each biopsy core a separate Gleason score. ${ }^{12}$ In the setting of more than two positive biopsy sites, pathologists usually grade the tumor at each site separately, and the Gleason score may differ from each positive site. This study seeks to determine if the highest Gleason score in all biopsy sites, or the Gleason score in the site with the highest tumor volume on the needle biopsy is the best predictor of final primary and secondary Gleason pattern grades in the radical prostatectomy specimens.

\section{Materials and methods}

\section{Biopsy Specimens}

This study analyzed 151 patients who underwent radical prostatectomy for prostate cancer between 1999 and 2003. The patients' ages ranged from age 41 to 77 years (median, 60 years). All had clinically localized cancer. None had hormonal or radiotherapy prior to surgery. Three patients had lymph node metastasis. Biopsies were preformed using an 18-gauge needle. All patients had positive needle biopsies in at least two biopsy sites. The biopsies were graded using the Gleason grading system. ${ }^{5}$ Various preoperative biopsy findings were recorded, including the primary and secondary Gleason pattern grade from each positive biopsy site, the highest Gleason score among all biopsy sites, and the Gleason score from the site with the highest tumor volume on the needle biopsy. Tumor volume in a positive biopsy site was defined as the percentage of areas of biopsy cores involved by cancer and was estimated visually, using 5\% increments.

This research was approved by the Indiana University Institutional Review Board.

\section{Radical Prostatectomy Specimens}

The radical prostatectomy specimens were examined in the whole-mount method as previously described. ${ }^{15-23}$ Prostates were weighed, measured, inked, and fixed in 10\% neutral formalin. Following fixation, the apex and base were amputated and serially sectioned at $3-5 \mathrm{~mm}$ intervals in the vertical, parasaggital plane. The seminal vesicles were sectioned parallel to their junction with the prostate and entirely submitted for examination. The remaining prostate was serially sectioned perpendicular to the long axis from the apex of the prostate to the base, and whole-mount sections were prepared.

The prostatectomy specimens were graded and staged by a single urologic pathologist (LC). Gleason score was obtained by the summation of primary Gleason pattern grade and secondary Gleason pattern grade based on the assessment of the entire specimen. The 1997 TNM (tumor, nodes, metastasis) system was used for pathologic staging. ${ }^{24}$ Surgical margins were considered positive when carcinoma cells were in contact with the inked margin. ${ }^{15}$ The volume of carcinoma in the entire prostate was determined by the grid method ${ }^{15,16,25-29}$ and was the sum of the volumes of individual foci of tumor. Patient and specimen characteristics are shown in Table 1.

\section{Statistical Analysis}

Spearman correlation coefficients were calculated between the Gleason grades from the biopsy and the Gleason grades in the radical prostatectomy specimen. To determine whether the Gleason score from the biopsy site with the highest Gleason score or the Gleason score from the biopsy site with the largest tumor volume on the needle biopsy was more correlated with the Gleason score and grades from the radical prostatectomy, the correlations were compared. Since the correlations are both calculated from the same sample, the method described in

Table 1 Characteristics of 151 patients who underwent radical prostatectomy for prostate cancer

\begin{tabular}{lc}
\hline Characteristic (n=151) & N (\%) or median (range) \\
\hline Age (years) & $60(41-77)$ \\
Preoperative PSA (ng/ml) & $5.7(0.3-48.7)$ \\
Prostate weight (g) & $35(14-105)$ \\
Primary Gleason grade (G1) & \\
2 & $8(5.3 \%)$ \\
3 & $108(71.5 \%)$ \\
4 & $28(18.5 \%)$ \\
5 & $7(4.7 \%)$ \\
& \\
Secondary Gleason grade (G2) & \\
2 & $15(9.9 \%)$ \\
3 & $61(40.4 \%)$ \\
4 & $64(42.4 \%)$ \\
5 & $11(7.3 \%)$ \\
Gleason score & \\
5 & $23(15.2 \%)$ \\
6 & $32(21.2 \%)$ \\
7 & $78(51.7 \%)$ \\
8 & $4(2.6 \%)$ \\
9 & $14(9.3 \%)$ \\
Pathologic stage & \\
T2a & \\
T2b & $16(10.6 \%)$ \\
T3a & $82(54.3 \%)$ \\
T3b & $44(29.1 \%)$ \\
Tumor volume (ml) & $9(6 \%)$ \\
Lymph node metastasis (positive) & \\
Surgical margins (positive) & \\
Extraprostatic extension (positive) & $2.1(0.3-13.6)$ \\
Seminal vesicle invasion (positive) & $30(26.5 \%)$ \\
High-grade PIN (positive) & $52(34.4 \%)$ \\
Multifocality of cancer (positive) & $149(99.3 \%)$ \\
& $131(86.8 \%)$ \\
\hline & \\
& \\
& \\
& \\
&
\end{tabular}


Cohen and Cohen, ${ }^{30}$ which takes their dependence into account was used to compare the correlation coefficients. The Wilcoxon Rank Sum test was used to test for associations between Gleason pattern grades and categorical variables.

\section{Results}

The distribution of biopsies are shown in Table 2 . The number of biopsies ranged from 2 to 12, with the majority of patients ( $42 \%$ ) having two biopsies. All of these patients had more than one positive biopsy site, and the number of positive biopsy sites ranged from 2 to 8 , with the majority $(68 \%)$ having two positive biopsy sites (Table 2). In total, 88 (58\%) patients had identical primary and secondary Gleason pattern grade from all positive sites. A total of $126(83 \%)$ patients had identical primary Gleason pattern grade from all positive sites. In all, 98 (65\%) patients had identical secondary Gleason pattern grade from all positive sites.

Table 3 contains the distribution of Gleason scores in needle biopsy and radical prostatectomy specimens. The primary Gleason pattern grade from the biopsy site with the highest Gleason score correlated exactly with primary Gleason pattern grade in the radical prostatectomy specimen in $75.5 \%$ of cases while it undergraded the primary Gleason pattern

Table 2 Distribution of biopsies in 151 prostate cancer patients who underwent radical prostatectomy

\begin{tabular}{lccc}
\hline $\begin{array}{l}\text { Number of biopsy } \\
\text { sites }\end{array}$ & $\mathrm{N}(\%)$ & $\begin{array}{c}\text { Number of positive } \\
\text { biopsy sites }\end{array}$ & $\mathrm{N}(\%)$ \\
\hline 2 & $63(42.0)$ & 2 & $102(67.6)$ \\
3 & $7(4.7)$ & 3 & $30(19.9)$ \\
4 & $22(14.7)$ & 4 & $10(6.6)$ \\
5 & $6(4.0)$ & 5 & $4(2.6)$ \\
6 & $33(22.0)$ & 6 & $2(1.3)$ \\
7 & $7(4.7)$ & 7 & $1(0.7)$ \\
8 & $9(6.0)$ & 8 & $2(1.3)$ \\
9 & 0 & 9 & 0 \\
10 & $1(0.7)$ & 10 & 0 \\
11 & 0 & 11 & 0 \\
12 & $2(1.3)$ & 12 & 0 \\
\hline
\end{tabular}

Table 3 Distribution of Gleason scores in needle biopsy and radical prostatectomy specimens $(N=151)$

\begin{tabular}{lccc}
\hline $\begin{array}{l}\text { Gleason } \\
\text { score }\end{array}$ & $\begin{array}{c}\text { Gleason score } \\
\text { from radical } \\
\text { prostatectomy } \\
\text { N (\%) }\end{array}$ & $\begin{array}{c}\text { Maximum } \\
\text { Gleason score } \\
\text { from biopsy } \\
\text { N (\%) }\end{array}$ & $\begin{array}{c}\text { Gleason score from } \\
\text { site with largest } \\
\text { tumor volume } \\
\text { N (\%) }\end{array}$ \\
\hline 4 & 0 & 0 & $2(1.3)$ \\
5 & $23(15.2)$ & $1(0.7)$ & $2(1.3)$ \\
6 & $32(21.2)$ & $61(40.4)$ & $66(43.7)$ \\
7 & $78(51.7)$ & $70(46.3)$ & $64(42.4)$ \\
8 & $4(2.6)$ & $11(7.3)$ & $11(7.3)$ \\
9 & $14(9.3)$ & $6(4.0)$ & $5(3.3)$ \\
10 & 0 & $2(1.3)$ & $1(0.7)$ \\
\hline
\end{tabular}

grade in $13.9 \%$ of cases and overgraded it in $10.6 \%$ of cases (Figure 1). The Gleason score from the biopsy site with the highest tumor volume on the needle biopsy also correlated exactly with primary Gleason pattern grade in the radical prostatectomy specimen in $75.5 \%$ of cases while it undergraded the primary grade in $12.6 \%$ of cases and overgraded it in $11.9 \%$ of cases (Figure 1). Overall, the primary Gleason pattern grade from both the biopsy site with the highest Gleason score and the biopsy site with the highest tumor volume on the needle biopsy correlated significantly $(P<0.001$ for both $)$ and equally well with final primary Gleason pattern grade at radical prostatectomy (Spearman correlation coefficient $=0.53$ for both, $P=0.90$ ).

The secondary Gleason pattern grade from the biopsy site with the highest Gleason score correlated exactly with secondary Gleason pattern grade in the radical prostatectomy specimen in $43.7 \%$ of cases, while it undergraded the secondary pattern grade in $30.5 \%$ of cases and overgraded it in $25.8 \%$ of cases (Figure 2). The secondary Gleason pattern grade from the biopsy site with the highest tumor volume on the needle biopsy correlated exactly with secondary Gleason pattern grade in the radical prostatectomy specimen in $47.7 \%$ of cases, while it undergraded the secondary Gleason pattern grade in $23.8 \%$ of cases and overgraded it in $28.5 \%$ of cases (Figure 2). The secondary Gleason pattern grade from both the biopsy site with the highest Gleason score and the biopsy site with the highest tumor volume on the needle biopsy correlated significantly $(P<0.001$ for both) with final secondary Gleason pattern grade at radical prostatectomy, although secondary Gleason pattern grade from the biopsy site with the highest tumor volume on the needle biopsy correlated with secondary Gleason pattern grade at radical prostatectomy marginally better than those from the biopsy site with the highest

\section{PRIMARY GLEASON GRADE}

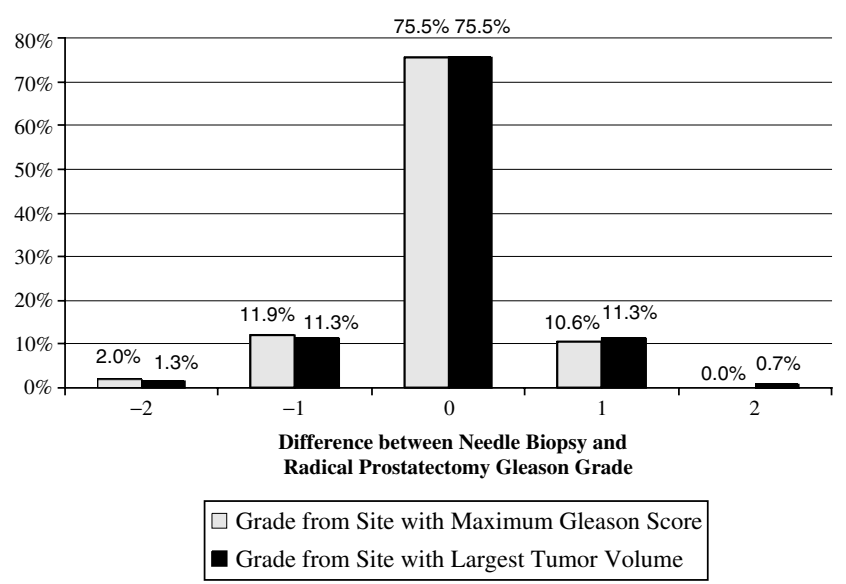

Figure 1 Correlation of primary Gleason pattern grade from the core with the highest tumor volume on the needle biopsy and the core with the highest Gleason score with primary Gleason pattern grade in radical prostatectomy specimens. 
SECONDARY GLEASON GRADE

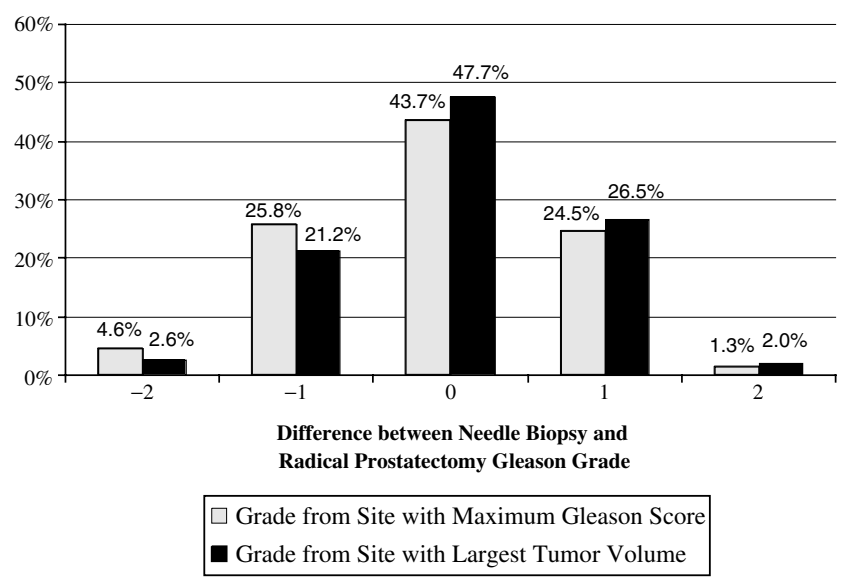

Figure 2 Correlation of secondary Gleason pattern grade from the core with the highest tumor volume on the needle biopsy and the core with the highest Gleason score with secondary Gleason pattern grade in radical prostatectomy specimens.

Gleason score (Spearman correlation coefficient, 0.32 vs $0.24 ; P=0.058$ ).

The Gleason score from both the biopsy site with the highest Gleason score and the biopsy site with the highest tumor volume on the needle biopsy correlated significantly $(P<0.001$ for both $)$ and equally well with the final Gleason score at radical prostatectomy (Spearman correlation coefficient $=0.54$ and 0.55 , respectively; $P=0.95$ ) (Figure 3 ). In all, 10 patients who had a Gleason score of 7 in the biopsy site with the highest Gleason score had a Gleason score of 6 in the radical prostatectomy, while a similar number of patients (9) who had Gleason score of 7 in the biopsy site had a Gleason score of 6 in the radical prostatectomy specimen.

Both maximum Gleason score from all biopsy sites and Gleason score from the site with the largest tumor volume on the needle biopsy were associated with final pathologic stage and extraprostatic extension $(P<0.001)$. Both maximum Gleason score from all biopsy sites and Gleason score from the site with the largest tumor volume on the needle biopsy were associated equally well with final pathologic stage and extraprostatic extension $(P<0.0001)$. Minimal correlation was found between biopsy Gleason score and patient's age, preoperative PSA levels, prostate weight, and tumor volume (all Spearman correlation coefficients $<0.23$ ). No significant association was found between biopsy Gleason score and extraprostatic extension, seminal vesicle invasion, surgical margin status, high-grade prostatic intraepithelial neoplasia, perineural invasion, or multifocality of cancer (all $P$-value $>0.05$ ).

\section{Discussion}

In this study, we found that the highest Gleason score from all biopsy sites and the Gleason score

\section{GLEASON SCORE}

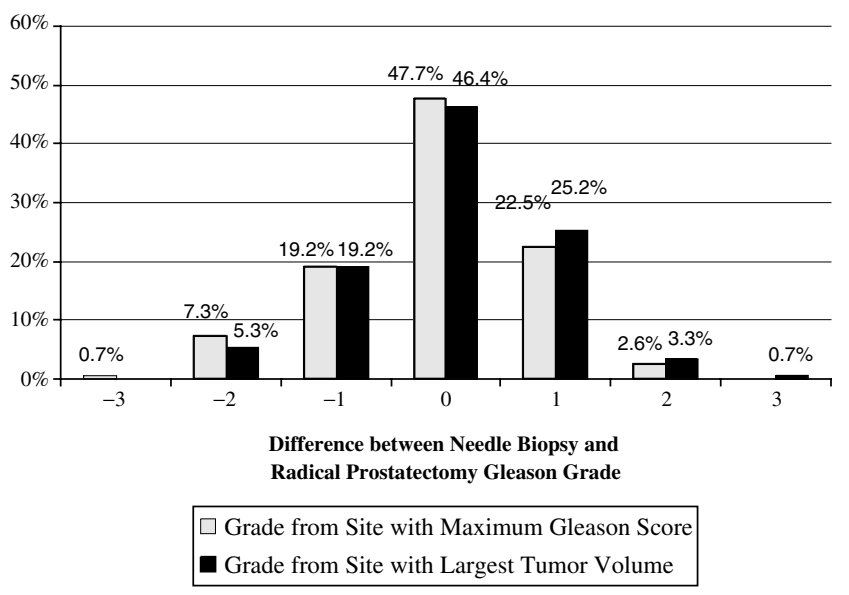

Figure 3 Correlation of overall Gleason score from the core with the highest tumor volume on the needle biopsy and the core with the highest Gleason score with final Gleason score in radical prostatectomy specimens.

from the site with the highest tumor volume on the needle biopsy are equally predictive of final Gleason score and primary Gleason pattern grade on radical prostatectomy. Both methods are predictive of secondary Gleason pattern grade on radical prostatectomy. We recommend that the highest Gleason score from all positive biopsy sites should be used when assigning an initial score using needle biopsies for improved accuracy and prognostic value.

The Gleason grading system is widely used in urologic pathology, has been adopted as a standard grading system by the 2004 World Health Organization (WHO) classification. ${ }^{31}$ The Gleason grading system is a powerful prognostic indicator for prostatic adenocarcinoma, validated by numerous studies. The prognostic power of the Gleason grading system, particularly the percentage of Gleason pattern grade $4 / 5$, in predicting PSA recurrence has been demonstrated by Stamey et $a l^{32}$ who showed this and intraprostatic vascular invasion to be the only variables predictive of PSA recurrence. The Gleason grading system does, however, have limitations, like any histologic grading system. ${ }^{33}$ Inter- and intraobserver reproducibility varies among pathologists. For example, Allsbrook et $a l^{1}$ have demonstrated only moderate reproducibility of grading among general pathologists and De Las Morenas et $a l^{34}$ have, in a comparison of the Gleason, Mostofi, and Böcking grading $\mathrm{s}$ ystems, found the Gleason system to be the least reproducible. These concerns about reproducibility are somewhat offset by other studies such as an additional study by Allsbrook et $a l^{1}$ that reveals good reproducibility of Gleason grading among urologic pathologists and a study by Renshaw et $a l^{35}$ that displays good concordance of Gleason grading between reference and general pathologists as well as demonstrating recent improvement in the 
reproducibility of grading prostatic adenocarcinomas among general pathologists. Another limitation of the Gleason grading system is well-documented tendency towards undergrading, particularly in needle biopsy. ${ }^{1-3,6,9,14}$ In addition, the difficulty remains in quantifying the percentages of Gleason pattern grades 3 and 4 when admixed, which could render the clinically significant distinction between a Gleason score of $3+4$ and $4+3$ difficult. $^{36}$

The ability of biopsy Gleason score to predict final Gleason score in the radical prostatectomy specimen has been studied previously. Bostwick ${ }^{2}$ studied 316 radical prostatectomies and found that the primary Gleason pattern grade on biopsy showed exact correlation with radical prostatectomy score in $54 \%$ of cases. This study showed $40 \%$ underestimation of prostatectomy Gleason score by biopsies and $25 \%$ overestimation, with the grading errors being more frequent in the lowest grade biopsies where there was a biopsy grading error of $91 \%$ in well differentiated tumors. ${ }^{2}$ The study by Cookson et $a l^{9}$ of 226 consecutive cases produced similar results with exact correlation of biopsy and prostatectomy scores in $31 \%$ of cases, overestimation of score in $15 \%$, and underestimation of final score in $54 \%$ of cases. Spires et $a l^{6}$ studied 67 patients and found exact correlation between biopsy and prostatectomy Gleason scores in $58 \%$ of cases. Carlson et $a l^{3}$ studied 106 patients, and documented similar results, with $68 \%$ exact correlation, $25 \%$ underestimation of prostatectomy score, and $8 \%$ overestimation of prostatectomy score. Steinberg et $a l^{14}$ studied 499 patients and found exact correlation in $58 \%$ of biopsy and radical prostatectomy specimens (when graded by pathologists at John's Hopkins Hospital). Steinberg et $a 1^{14}$ also found that a biopsy Gleason score of less than 7 predicted a radical prostatectomy Gleason score of less than 7,64\% of cases while a biopsy Gleason score of greater than (or equal to) 7 was predictive of a prostatectomy score greater than $7,88 \%$ of the time; again, demonstrating the problem of underestimation of grade.

Clinicians routinely use sextant biopsies to detect cancer. In many cases, there are multiple positive biopsy sites, and current recommendations are to assign each biopsy a separate score. ${ }^{12}$ If there are more than two positive sites, each will be assigned a separate score, which may differ from site to site. This study was specifically designed to answer the question of, in the setting of multiple positive biopsy sites, which is the best predictor of final Gleason score. We found that the ability of both the Gleason score from the site with the highest tumor volume on the needle biopsy and the highest Gleason score from all sites to predict radical prostatectomy Gleason scores were comparable to those of techniques used in other studies with only a single positive score. Primary Gleason grade was predicted exactly in $75.5 \%$ of cases using either method in this study. Our data also show trends that both techniques of Gleason grade prediction more commonly underestimate Gleason score than they overestimate the score, similar trends were reported in other studies. ${ }^{1-4,6,9,14,37}$ The origin of this type of error is likely due to sampling issues and tumor heterogeneity, although pathologic grading errors, borderline cases, and reverse sampling errors may also contribute to this discrepancy. ${ }^{14}$

We also found that secondary Gleason pattern grade from the biopsy site with the highest tumor volume on the needle biopsy correlated with secondary Gleason pattern grade at radical prostatectomy marginally better than those from the biopsy site with the highest Gleason score (Spearman correlation coefficient, 0.32 vs $0.24, P=0.058$ ). This may be related to the so-called reverse sampling error. ${ }^{1,14}$ In this form of error, a minor component of tumor is sampled in needle biopsy and included in calculating the biopsy-derived Gleason score, but comprises $<5 \%$ of the entire tumor in the radical prostatectomy specimen, so it is not included in the final grading since Gleason grading is based on the two most prevalent patterns observed. Reverse sampling error is used by Yang et $a l^{37}$ as a possible explanation of why small (less than $1.5 \mathrm{~mm}$ ) foci of Gleason score 7 carcinoma on needle biopsy are not an adverse finding. The elimination or reduced frequency of this form of error may improve accuracy of predicting final Gleason scores and Gleason pattern grades.

The significance of any high-grade cancer on biopsies should not be underestimated. Kunz and Epstein found that any Gleason $4+4=8$ (highgrade) tumor in biopsies is significant in terms of predicting both stage and radical prostatectomy grade, even if it is present in only one core, and greater numbers of cores have lower-grade tumor. ${ }^{12}$ Pan et $a l^{7}$ has shown that small tertiary foci of high-grade cancer in prostatectomy specimens are predictive of tumor aggressiveness, again suggesting that the presence of any high-grade tumor is significant. Studies by Bostwick, Carlson et al, Spires et al, and Steinberg et al, all have found that the volume of tumor present in biopsies does not correlate with a change in the ability to predict radical prostatectomy Gleason score, emphasizing the significance of small-volume, high-grade elements. ${ }^{2,3,6,14}$ Our data also indicate that both the highest Gleason score from all sites and the Gleason score from the site with the highest tumor volume on the needle biopsy are equally and significantly predictive of final Gleason score on radical prostatectomy. The reason that an increased volume of tumor on the needle biopsy is not more accurate in predicting final Gleason score may be attributed to the extremely small size of a biopsy as compared to a prostate (estimated to be approximately $1 / 10000$ th the size of a prostate $)^{1}$ and the sampling error inherent to biopsies.

In conclusion, in the setting of multiple positive prostate biopsy sites, both the Gleason score from 
the biopsy site with the highest tumor volume on the needle biopsy and from the site with the highest Gleason score of all sites are predictive of final scores and grades on radical prostatectomy, and both could be used in assigning an initial score using needle biopsies. We recommend that the highest Gleason score from all positive biopsy sites should be used for practical purpose.

\section{References}

1 Allsbrook WC, Mangold KA, Yang X, et al. The Gleason grading system. J Urol Pathol 1999;10:141-157.

2 Bostwick DG. Gleason grading of prostatic needle biopsies. Am J Surg Pathol 1994;18:796-803.

3 Carlson GD, Calvanese CB, Kahane H, et al. Accuracy of biopsy Gleason scores from a large uropathology laboratory: use of a diagnostic protocol to minimize observer variablity. Urology 1998;51:525-529.

4 Fernandes ET, Sundaram CP, Long R, et al. Biopsy Gleason score: how does it correlate with the final pathologic diagnosis in prostate cancer? Br J Urol 1997;79:615-617.

5 Gleason DF, Mellinger GT. Prediction of prognosis for prostatic adenocarcinoma by combined histological grading and clinical staging. Veterans administration cooperative urologic research group. J Urol 1974;111: 58-64.

6 Spires SE, Cibull ML, Wood DP, et al. Gleason histologic grading in prostatic carcinoma. Arch Pathol Lab Med 1994;118:705-708.

7 Pan C, Potter SR, Partin AW, et al. The prognostic significance of tertiary Gleason patterns of higher grade in radical prostatectomy specimens: a proposal to modify the Gleason grading system. Am J Surg Pathol 2000;24:563-569.

8 Bostwick DG, Qian J, Schlesinger C. Contemporary pathology of prostate cancer. Urol Clin N Am 2003; 30:181-207.

9 Cookson MS, Fleshner NE, Soloway SM, et al. Correlation between Gleason score of needle biopsy and radical prostatectomy specimens: sccuracy and clinical implications. J Urol 1997;157:559-562.

10 Epstein JI. Gleason score 2-4 adenocarcinoma of the prostate on needle biopsy: a diagnosis that should not be made [Letter]. Am J Surg Pathol 2000;24:477-478.

11 Epstein JI, Potter SR. The pathological interpretation and significance of prostate needle biopsy findings: mplications and current controversies. J Urol 2001; 166:402-410.

12 Kunz GM, Epstein JI. Should each core with prostate carcinoma be assigned a separate gleason score? Hum Pathol 2003;34:911-914.

13 Noguchi M, Stamey TA, McNeal JE, et al. Relationship between systemic biopsies and histologic features of 122 radical prostatectomy specimens: lack of prediction of tumor significance for men with nonpalpable prostate cancer. J Urol 2001;166:104-110.

14 Steinberg DM, Sauvageot J, Piantadosi S, et al. Correlation of prostate needle biopsy and radical prostatectomy Gleason grade in academic and community settings. Am J Surg Pathol 1997;21:566-567.

15 Cheng L, Darson MF, Bergatrahl EJ, et al. Correlation of margin status and extraprostatic extension with progression of prostate carcinoma. Cancer 1999;86: 1775-1781.

16 Cheng L, Slezak J, Bergstralh EJ, et al. Preoperative prediction of surgical margin status in prostate cancer patients treated by radical prostatectomy. J Clin Oncol 2000;18:2862-2868.

17 Bostwick DG, Myers RP, Oesterling JE. Staging of prostate cancer. Semin Surg Oncol 1994;10:60-72.

18 Poulos CK, Daggy JK, Cheng L. Prostate needle biopsies: multiple variables are predictive of final tumor volume in radical prostatectomy specimens. Cancer 2004;101:527-532.

19 Eichelberger LE, Cheng L. Does pT2b prostate carcinoma exist? Critical appraisal of the 2002 TNM classification of prostate carcinoma. Cancer 2004;100: 2573-2576.

20 Arora R, Koch MO, Eble JN, et al. Heterogeneity of Gleason grade in multifocal adenocarcinoma of the prostate. Cancer 2004;100:2362-2366.

21 Ng JC, Koch MO, Daggy JK, et al. Perineural invasion in radical prostatectomy specimens. J Urol (in press).

22 Poulos CK, Koch MO, Eble JN, et al. Bladder neck invasion is an independent predictor of prostate specific antigen recurrence. Cancer 2004;101:15631568.

23 Emerson RE, Koch MO, Daggy J, et al. Closest distance between tumor and resection margin in radical prostatectomy specimens: lack of prognostic significance. Am J Surg Pathol (in press).

24 Fleming ID, Cooper JS, Henson DE, et al. AJCC Cancer Staging Manual. Philadelphia: Lippincott Raven, 1997.

25 Humphrey PA, Vollmer RT. Intraglandular tumor extent and prognosis in prostatic carcinoma: application of a grid method to prostatectomy specimens. Hum Pathol 1990;21:799-804.

26 Qian J, Wollan P, Bostwick DG. The extent and multicentricity of high grade prostatic intraepithelial neoplasia in clinically localized prostatic adenocarcinoma. Hum Pathol 1997;28:143-148.

27 Dugan JA, Bostwick DG, Myers RP, et al. The definition and preoperative prediction of clinically insignificant prostate cancer. JAMA 1996;275:288-294.

28 Eichelberger LE, Koch MO, Daggy JK, et al. Predicting tumor volume in radical prostatectomy specimens from patients with prostate cancer. Am J Clin Pathol 2003;120:386-391.

29 Hong H, Koch MO, Foster RS, et al. Anatomic distribution of periprostatic adipose tissue: a mapping study of 100 radical prostatectomy specimens. Cancer 2003;97:1639-1643.

30 Cohen J, Cohen P. Applied Multiple Regression/ Correlation Analysis for the Behavioral Sciences. Hillsdale, NJ: Lawrence Erlbaum Associates, 1983.

31 Eble JN, Epstein JI, Sauter G, et al. WHO Classfication of Tumours: Pathology and Genetics. Tumours of the Urinary and Male Reproductive System. IARC Press: Lyon, France, 2004.

32 Stamey T, McNeal J, Yemoto C, et al. Biological determinants of cancer progression in men with prostate cancer. JAMA 1999;281:1395-1400.

33 Humphrey PA. Gleason grading and prognostic factors in carcinoma of the prostate. Mod Pathol 2004;17: 292-306.

34 De Las Morenas A, Siroky R, Merriam J, et al. Prostatic adenocarcinoma: reproducibility and correlation with clinical stages of four grading systems. Hum Pathol 1988;19:595-597. 
35 Renshaw AA, Schultz D, Cote K, et al. Accurate gleason grading of prostatic adenocarcinoma in prostate needle biopsies by general pathologists. Arch Pathol Lab Med 2003;27:1007-1008.

36 Chan TY, Partin AW, Walsh PC, et al. Prognostic significance of Gleason score 3+4 versus gleason score
$4+3$ tumor at radical prostatectomy. Urology 2000; 823-827.

37 Yang XJ, Lecksell K, Potter SR, et al. Significance of small foci of Gleason score 7 or greater prostate cancer on needle biopsy. Urology 1999;54: 528-532. 